\title{
Perfil de diagnósticos de enfermagem antes de iniciar o tratamento hemodialítico*
}

\author{
Profile of nursing diagnoses before to start the hemodialitic treatment \\ Perfíl de diagnósticos de enfermería antes del inicio del tratamiento con la hemodiálisis
}

\author{
Mariane Muniz Bisca', Isaac Rosa MarQues' \\ 'Universidade de Santo Amaro. Curso de Enfermagem. São Paulo, SP
}

Submissão: 17/12/2009

Aprovação: 03/05/2010

\section{RESUMO}

Tratou-se de um estudo inter-relacional retrospectivo,o Qual teve como objetivo descrever o perfil dos diagnósticos de enfermagem de 31 pacientes em início de tratamento hemodialítico em uma clínica de hemodiálise na Zona Sul de São Paulo. A pesquisa foi realizada a partir de prontuários. Os dados utilizados incluíram: identificação do paciente, doença de base, dados do exame físico, anotações e evoluções multiprofissionais. Os diagnósticos de enfermagem mais prevalentes incluíram: perfusão tissular renal ineficaz (100,0\%), risco para infecção (100,0\%), proteção ineficaz $(80,6 \%)$ e controle ineficaz do regime terapêutico $(54,8 \%)$. Concluiu-se Que a identificação do perfil de diagnósticos destes pacientes é essencial para o planejamento da assistência de enfermagem após o início do programa de hemodiálise.

Descritores: Insuficiência renal; Diálise renal; Diagnósticos de enfermagem; Enfermagem.

ABSTRACT
This was a retrospective study, which aimed to describe the profile of nursing diagnoses of 31 patients starting hemodialysis treatment in a hemodialysis clinic in the South Zone of São Paulo. The survey was conducted from patients' records. The data used included: patient identification, underlying disease, data from physical examination notes and multidisciplinary evaluations. The most prevalent nursing diagnoses included: ineffective renal tissue perfusion (100.0\%), risk for infection (100.0\%), innefective protection (80.6\%) and ineffective management of therapeutic regimen (54.8\%). It was concluded that the identification of the profile of nursing diagnoses of these patients is essential for the planning of nursing care after initiation of hemodialysis.

Key words: Renal insufficiency; Renal dialysis; Nursing diagnoses; Nursing.

\section{RESUMEN}

El presente estudio retrospectivo tuvo como objetivo describir el perfil de los diagnósticos de enfermería de 31 pacientes Que iniciaron tratamiento de hemodiálisis en una clínica de hemodiálisis en la Zona Sur de São Paulo. La investigación fue realizada a partir de registros de los pacientes. Los datos utilizados fueron: la identificación del paciente, enfermedad de base, los datos de las notas del examen físico y evaluaciones multidisciplinarias. Los diagnósticos de enfermería más frecuentes fueron: perfusión tisular inefectiva renal (100,0\%), riesgo de infección (100,0\%), protección ineficaz $(80,6 \%)$ y control ineficaz del régimen terapéutico $(54,8 \%)$. Se concluyó Que la identificación del perfil de los diagnósticos de enfermería de estos pacientes es esencial para la planificación de cuidados de enfermería tras el inicio de la hemodiálisis.

Descriptores: Insuficiencia renal; Diálisis renal; Diagnósticos de enfermería; Enfermagem.

*Trabalho de Conclusão de Curso apresentado ao Curso de Enfermagem da Universidade de Santo Amaro, em dezembro de 2009.

AUTOR CORRESPONDENTE Mariane Muniz Bisca. Rua Laudelino dos Santos, 44. Vila São ludas. CEP 04930-280. São Paulo, SP.

E-mail: mariane_muniz30@yahoo.com.br 


\section{INTRODUÇÃO}

A Doença Renal Crônica (DRC) consiste da perda progressiva e irreversível das funções renais Que pode iniciar com um Quadro agudo ou de maneira lenta e progressiva. O tratamento definitivo indicado é o transplante renal. Trata-se de um processo moroso, e até Que ele se concretize, a única alternativa para manter a vida esta no tratamento dialítico contínuo Que compreende duas modalidades: diálise peritonial ou hemodiálise. A maioria das pessoas com IRC, no Brasil segue programas de hemodiálise ambulatorial realizados em regime de três vezes semanais com duração de, aproximadamente, três a Quatro horas para cada sessão. A hemodiálise substitui a função renal pelo processo de remoção de tóxicos e outras substâncias nocivas ao organismo, através de uma circulação sanguínea extra-corpórea ${ }^{(1)}$.

A hemodiálise na maioria das vezes representa uma esperança de vida, já Que a doença é um processo irreversível. Contudo observa-se Que geralmente as dificuldades de adesão ao tratamento estão relacionadas a não aceitação da doença, à percepção de si próprio e ao relacionamento interpessoal com familiares e ao convívio social( ${ }^{(2)}$.

O agir e o pensar relacionados à assistência ao ser humano devem ser prioridades na área da saúde, principalmente para a enfermagem Que tem o cuidar como um dos elementos essenciais de sua prática ${ }^{(3)}$.

O enfermeiro como coordenador da equipe deve coordenar a assistência prestada, identificando as necessidades individuais de cada cliente, proporcionando meios de atendimento Que visem uma melhor adeQuação do tratamento, garantindo assim uma Qualidade de vida melhor, aproveitando todos os momentos para criar condições de mudanças Quando necessário. A prática do cuidar personalizado está diretamente ligada à Qualidade da assistência prestada, e uma das formas de alcançar este objetivo é através do processo de enfermagem ${ }^{(2)}$.

A responsabilidade do cuidar exige Que todas as intervenções propostas sejam fundamentadas na avaliação do estado de saúde do indivíduo requerendo que se adote o diagnóstico de enfermagem como referência ${ }^{(4)}$.

Com o levantamento dos principais problemas Que o paciente em hemodiálise apresenta e a atribuição dos diagnósticos de enfermagem torna mais fácil direcionar a assistência e enxergar o paciente de maneira completa ${ }^{(5)}$.

O diagnóstico de enfermagem é uma etapa Que se reveste de singular importância, pois fornece meios para propor intervenções de responsabilidade exclusiva do enfermeiro Quanto aos problemas de saúde detectados. O estudo desse diagnóstico proporciona ainda o uso de uma linguagem própria do enfermeiro facilitando a comunicação com os pacientes ${ }^{(3)}$.

Considerando este contexto, o estudo teve como objetivo identificar e descrever o perfil de diagnósticos de enfermagem do paciente renal crônico em início de tratamento hemodialítico em uma unidade de hemodiálise da cidade de São Paulo.

\section{METODOLOGIA}

Tratou-se de um estudo ${ }^{(6)}$, baseado na consulta de dados existentes em prontuário do paciente.
A pesquisa foi realizada em uma clínica de hemodiálise no município de São Paulo, local escolhido devido à facilidade de acesso e ao grande fluxo de pacientes em tratamento hemodialítico ambulatorial. Como característica, esta unidade tem atendimento médio de 40 pacientes por turno de sessão de hemodiálise, sendo realizados três turnos por dia, totalizando 120 pacientes/dia Que se alternam (Seg/Qua/Sex e Ter/Qui/Sab).

$\mathrm{Na}$ admissão do paciente é aberto um prontuário eletrônico, constando a identificação do paciente, consulta médica e exame físico de admissão, evolução médica e de enfermagem e prescrição medicamentosa.

A amostra foi composta por prontuários de pacientes admitidos para tratamento hemodialítico no período de abril de 2008 a abril de 2009, Que estavam acessíveis nos registros eletrônicos e Que atenderam os critérios de inclusão: ficha de admissão com exame físico.

A técnica de amostragem foi do tipo não-probabilística por conveniência, considerando a totalidade dos prontuários existentes; ou seja, foram acessados os prontuários Que estavam acessíveis de acordo com os critérios apresentados anteriormente. Foram excluídos os prontuários de pacientes que já haviam iniciado a hemodiálise em outro serviço (constavam como transferência).

$\mathrm{O}$ instrumento de coleta de dados (anexo) constou dos seguintes dados: identificação do paciente (cor, estado civil, faixa etária, gênero e procedência), doenças de base e treze diagnósticos de enfermagem e suas características definidoras principais, de acordo com Souza et $\mathrm{al}^{(2)}$ e Lata et $\mathrm{al}^{(7)}$. A composição e definição dos diagnósticos foi baseada na Taxomonina II da North-American Nursing Diagnoses Association $^{(8)}$.

Os dados foram coletados durante o mês de maio de 2009 . Inicialmente os prontuários foram separados e posteriormente analisados de acordo com o período estabelecido e os critérios de inclusão. Os dados do prontuário foram recuperados por meio do sistema computadorizado existente na instituição onde o estudo foi realizado.

A análise dos dados consistiu do estabelecimento de estatística descritiva, com dados absolutos e relativos. A base da análise foi a verificação da ocorrência das características definidoras para a composição dos treze diagnósticos de enfermagem previamente sugeridos.

O projeto de pesquisa foi aprovado pelo Comitê de Ética em Pesquisa da Universidade de Santo Amaro sob o parecer número: 032/2009.

\section{RESULTADOS E DISCUSSÃO}

\section{Caracterização da Amostra}

No período de abril de 2008 a abril de 2009, 69 pacientes iniciaram tratamento hemodialítico na unidade. Destes, 31 eram pacientes Que estavam iniciando a Terapia Renal Substitutiva pela primeira vez. Os 38 restantes, eram pacientes Que já haviam iniciado a terapia em outras clínicas e foram excluídos do estudo.

Os prontuários dos 31 pacientes utilizados neste estudo foram avaliados Quanto às características demográficas. A faixa etária de maior frequência foi a de pacientes de 40 a 59 anos $(58,1 \%)$. Quanto ao gênero houve predominância de indivíduos do sexo masculino $(64,5 \%)$. Em relação a cor $61,3 \%$ dos indivíduos eram 
de cor branca, ao estado civil 77,4\% eram casados e a procedência 48,4\% eram naturais de São Paulo. Maiores detalhes na Tabela 1 .

Os resultados das variáveis idade e gênero assemelham-se aos dados obtidos no estudo de Mendonça e Lima ${ }^{(9)}$ realizado no estado de Goiás com 63 pacientes, no Qual a faixa etária predominante foi a de $40-59$ anos com $(44,5 \%)$ e em relação ao gênero $(55,5 \%)$ dos pacientes eram do sexo masculino.

Quanto às doenças de base para o desenvolvimento da Doença Renal Crônica, foi constatado Que 29,0\% dos pacientes apresentaram Hipertensão Arterial Sistêmica, 19,4\% Diabetes Mellitus, 22,6\% a associação das duas patologias. Outras moléstias também foram demonstradas como causa da DRC, sendo a Glomerulonefrite presente em 12,9\%, a Doença Renal Policística em 12,9\% e as Uropatias Obstrutivas em 3,2\% dos pacientes Que iniciaram a terapia renal substitutiva na unidade de hemodiálise onde o estudo foi realizado (Tabela 2).

Foi evidenciado neste trabalho Que a Hipertensão Arterial Sistêmica como principal causa para o desenvolvimento da DRC. Este resultado se aproxima ao estudo realizado por Godinho et $\mathrm{al}^{(10)}$ com 122 pacientes em um hospital em Salvador no Qual 41,0\% apresentaram HAS, 6,6\% Diabetes Mellitus e 27,9\% HAS associada a DM.

Segundo dados da Sociedade Brasileira de Nefrologia a hipertensão arterial e o diabetes são responsáveis por cerca de metade dos pacientes Que estão em tratamento dialítico no Brasil ${ }^{(11)}$.

\section{Diagnósticos de enfermagem}

Após a análise das informações contidas nos prontuários dos 31 pacientes foram descritos através do processo de raciocínio diagnóstico onze diagnósticos de enfermagem. Os diagnósticos encontrados estão apresentados na Tabela 3.

Observa-se que o diagnóstico de enfermagem "perfusão tissular ineficaz: renal" e "risco de infecção" estiveram presentes em 100\% dos prontuários analisados, en@uanto Que para " proteção ineficaz" e "controle ineficaz do regime terapêutico" a porcentagem foi de $80,6 \%$ e $54,8 \%$ respectivamente.

O diagnóstico de enfermagem "perfusão tissular ineficaz: renal" definido pela diminuição na oxigenação, resultando na incapacidade de nutrir os tecidos no nível capilar, tendo como característica definidora principal registrada a elevação das taxas de uréia e creatinina sanguíneas, estava associado à alta incidência de HAS na maioria dos pacientes com este diagnóstico médico ${ }^{(8)}$. A HAS é o maior causador de $\mathrm{DRC}^{(12)}$.

O diagnóstico de enfermagem "risco para infecção" é definido como risco aumentado de ser invadido por organismos patogênicos. Este diagnóstico esteve presente em 100\% dos prontuários analisados, confirmado através dos fatores de risco observados: doença crônica, defesas secundárias inadequadas e procedimentos invasivos $^{(8)}$. De acordo com Costa et $\mathrm{al}^{(13)}$ e Leal et $\mathrm{al}^{(14)}$ os estados metabólicos em constante alteração favorecem a instalação de infecções no portador de DRC.

Estes resultados são compatíveis ao estudo de Lata et $\mathrm{al}^{\left({ }^{(7)}\right.} \mathrm{com}$ vinte pacientes adultos em tratamento hemodialítico em uma clínica no Rio de Janeiro, no Qual foram identificados em todos os pacientes os diagnósticos de perfusão tissular ineficaz renal e risco para infecção.

O diagnóstico de enfermagem "proteção ineficaz" é definido
Tabela 1. Características demográficas dos pacientes Que iniciaram tratamento hemodialítico. São Paulo, 2009.

\begin{tabular}{|c|c|c|}
\hline Variáveis & $\mathrm{n}$ & $\%$ \\
\hline \multicolumn{3}{|l|}{ Idade (em anos) } \\
\hline De 20 a 39 & 5 & 16,1 \\
\hline De 40 a 59 & 18 & 58,1 \\
\hline$>60$ & 8 & 25,8 \\
\hline \multicolumn{3}{|l|}{ Gênero } \\
\hline Feminino & 11 & 35,5 \\
\hline Masculino & 20 & 64,5 \\
\hline \multicolumn{3}{|l|}{ Cor } \\
\hline Branca & 19 & 61,3 \\
\hline Negro & 5 & 16,1 \\
\hline Outra & 7 & 22,6 \\
\hline \multicolumn{3}{|l|}{ Estado Civil } \\
\hline Solteiro & 2 & 6,5 \\
\hline Casado & 24 & 77,4 \\
\hline Divorciado & & \\
\hline Viúvo & 5 & 16,1 \\
\hline \multicolumn{3}{|l|}{ Procedência } \\
\hline São Paulo & 15 & 48,4 \\
\hline Bahia & 8 & 25,8 \\
\hline Minas Gerais & 3 & 9,7 \\
\hline Outros & 5 & 16,1 \\
\hline
\end{tabular}

Tabela 2. Doenças de base como principais causas para o desenvolvimento da Doença Renal Crônica. São Paulo, 2009.

\begin{tabular}{llc}
\hline Doença de Base & $\mathbf{n}$ & \% \\
\hline Hipertensão Arterial Sistêmica & 9 & 29,0 \\
Diabetes Mellitus & 6 & 19,4 \\
Doença Renal Policística & 4 & 12,9 \\
Glomerulonefrite & 4 & 12,9 \\
HAS e DM & 7 & 22,6 \\
Uropatias Obstrutivas & 1 & 3,2 \\
\hline
\end{tabular}

Tabela 3. Diagnósticos de enfermagem identificados nos pacientes com DRC em início de tratamento hemodialítico em uma clínica de hemodiálise na cidade de São Paulo. São Paulo, 2009.

\begin{tabular}{lcc}
\hline Diagnósticos de Enfermagem & $\mathbf{n}$ & \% \\
\hline Ansiedade & 3 & 9,6 \\
Controle ineficaz do regime terapêutico & 17 & 54,8 \\
Dor aguda & 8 & 25,8 \\
Eliminação urinária prejudicada & 6 & 19,4 \\
Intolerância a atividade & 7 & 22,6 \\
Náusea & 5 & 16,1 \\
Nutrição deseQuilibrada: menos do Que & & \\
as necessidades corporais & 10 & 32,2 \\
Perfusão Tissular Ineficaz: Renal & 31 & 100,0 \\
Proteção Ineficaz & 25 & 80,6 \\
Risco para infecção & 31 & 100,0 \\
Volume excessivo de líquidos & 16 & 51,6 \\
\hline
\end{tabular}

pela diminuição na capacidade de proteger-se de ameaças internas ou externas, como doenças ou lesões, caracterizadas na amostra por anorexia, dispnéia, fadiga e fraqueza, relacionado a perfis 
sanguíneos anormais (anemia) ${ }^{(8)}$, foi evidenciado em $80,6 \%$ dos prontuários da amostra. De acordo com Abensur et $\mathrm{al}^{(15)}$ a anemia é uma complicação da doença renal crônica, tendo diversas causas, sendo a deficiência relativa de eritropoetina e a carência de ferro as mais importantes. A anemia provoca palidez cutânea, fraqueza, indisposição, déficit de atenção, prejuízo na Qualidade de vida e maior mortalidade nos pacientes com doença renal crônica.

O diagnóstico de enfermagem "controle ineficaz do regime terapêutico" é um padrão de regulação e interação à vida diária de um programa de tratamento de doenças e suas sequelas que é insatisfatório para atingir objetivos de saúde ${ }^{(8)}$. Este diagnóstico foi constatado em $54,8 \%$ dos prontuários analisados e está relacionado ao déficit de conhecimento da doença, caracterizado pelo uso irregular das medicações. Segundo Bastos et $\mathrm{a}^{(12)}$, permanecer no regime terapêutico é uma das grandes dificuldades do portador de DRC. Segundo estes autores, os fatores socioeconômicos e o desconhecimento do processo patológico em si, podem acelerar o curso da doença. Em alguns casos, a falta de apoio familiar para procurar o devido atendimento médico pode também contribuir para a não aderência ao regime terapêutico ${ }^{(16)}$.

O diagnóstico de enfermagem "volume excessivo de líquidos" foi identificado em $51,6 \%$ dos prontuários analisados, é definido por "retenção aumentada de líeuidos isotônicos", sendo anasarca, azotemia, congestão pulmonar, dispnéia, edema, oligúria, ortopnéia e ruídos respiratórios adventícios as características definidoras mais evidenciadas na amostra ${ }^{(8)}$.

O diagnóstico de enfermagem "nutrição desequilibrada: menos do Que as necessidades corporais" foi evidenciado em 32,2\% dos prontuários. A desnutrição acomete o portador de DRC devido a vários fatores. Segundo Valenzuela et $\mathrm{al}^{(17)}$, a desnutrição desempenha um importante impacto sobre a morbidade e mortalidade de pacientes Que venham a ser submetidos a hemodiálise. Os pacientes portadores de Diabetes Mellitus ${ }^{(1)}$, sobretudo, são os Que apresentam maior risco de desnutrição. A desnutrição ainda tem correlação com a eficiência da hemodiálise ${ }^{(18)}$.

O diagnóstico de enfermagem "dor aguda" é caracterizado basicamente pelo relato verbal de dor ${ }^{(8)}$. Este diagnóstico foi identificado em $25,8 \%$ dos prontuários analisados. A Qualidade de vida do portador de DRC é altamente influenciada pelas sensações de dor. Um estudo demonstrou que a dor está presente em 59 a $61 \%$ dos pacientes adultos portadores de $\mathrm{DRC}^{(19)}$. A dor é um componente de restrição física Que influencia na demanda ocupacional como comorbidades, sendo fator-chave para prejuízos sociais ${ }^{(20)}$.

O diagnóstico de enfermagem "intolerância à atividade", é caracterizado pela energia fisiológica insuficiente para suportar ou completar as atividades diárias requeridas ou desejadas, caracterizado por desconforto aos esforços, dispnéia aos esforços e relato verbal de fadiga ou fraqueza ${ }^{(8)}$, sendo identificado em $22,6 \%$ da amostra. Segundo Kusumota et $\mathrm{al}^{(2 \mathrm{l})}$, esta é uma alteração bastante frequente em pacientes com DRC, sobretudo nos idosos. Isto se deve ao comprometimento cardíaco em manipular a sobrecarga hídrica, acarretando congestão pulmonar.

O diagnóstico de enfermagem "eliminação urinária prejudicada" é definido pelo distúrbio na eliminação da urina ${ }^{(8)}$, e esteve presente em 19,4\% da amostra, caracterizado por noctúria, retenção urinária e urgência urinária.

O diagnóstico de enfermagem "náusea" foi identificado em 16, 1\% dos prontuários, tendo como característica principal o relato verbal de náusea ${ }^{(8)}$.

As características da DRC e seus tratamentos favorecem o aparecimento da ansiedade devido à presença constante de situações ameaçadoras Que os doentes enfrentam. Entre os fatores Que favorecem o surgimento da ansiedade nos pacientes em hemodiálise destacam os seguintes: restrição da dieta, diminuição da capacidade sexual, mudanças nos relacionamentos sociais e familiares, mudança na aparência física, medo do desconhecido e medo da morte ${ }^{(2)}$.

Neste estudo o diagnóstico de enfermagem "ansiedade" esteve presente em 9,6\% da amostra, dados Que se assemelham aos encontrados no estudo realizado por Souza et $\mathrm{al}^{(2)}$, no Qual a porcentagem deste diagnóstico foi de 10\%.

\section{CONCLUSÃO}

Foram identificados e descritos onze diagnósticos de enfermagem a partir dos dados existentes nos prontuários. A identificação e descrição destes diagnósticos de enfermagem permitiram conhecer a situação inicial destes pacientes frente à DRC.

O diagnóstico de enfermagem, "controle ineficaz do regime terapêutico" é o Que mais chama a atenção. Grande parte dos pacientes evoluiu para piora da função renal devido o não seguimento de um regime terapêutico indicado. Isto ressalta a importância de Que a enfermagem deva reforçar os programas de educação sobre a terapia a ser iniciada. Caso este processo educativo não seja devidamente implementado é provável Que a hemodiálise não produza os efeitos desejados e o paciente não obtenha os benefícios da mesma.

A identificação deste perfil de diagnósticos é essencial para a etapa do planejamento da assistência de enfermagem. Os pacientes submetidos à terapia da hemodiálise necessitam de assistência individualizada e os diagnósticos de enfermagem são considerados como a base de todo o processo de enfermagem também. Planejar adequadamente as intervenções de enfermagem promove impacto sobre os resultados e também da Qualidade desta assistência.

Ao identificar estes diagnósticos, uma base de conhecimento foi estabelecida. Novos estudos para validação deste perfil são ainda necessários, considerando a limitação amostral. Novos estudos sobre intervenções de enfermagem são igualmente necessários para completar o presente estudo.

\section{REFERÊNCIAS}

I. Trentini M, Corradi EM, Araldi MAR, Tigrinho FC. Qualidade de vida de pessoas dependentes de hemodiálise considerando alguns aspectos físicos, sociais e emocionais. Texto Contexto Enferm 2004; 13(1); 74-82.
2. Souza EM, Martino MMF, Lopes MHBM. Diagnósticos de enfermagem em pacientes com tratamento hemodialítico utilizando o modelo teórico de Imogene King. Rev Esc Enferm USP 2007; 4I(4): 629-35. 
3. Lira ALBC, Albuquerque IG, Lopes MVO. Perfil dos Diagnósticos de Enfermagem em pacientes transplantados renais. Rev Enferm UERJ 2007; 15(9): 13-18

4. Braga CG, Cruz DALM. A taxonomia II proposta pela north american nursing diagnosis association (NANDA). Rev LatinoAm Enfermagem 2003; 1 I (2):240-4.

5. Domingos ACS, Ribeiro ACFS, Mota TC, Boaventura AP, Nascimento GA. Diagnósticos de enfermagem em uma unidade de hemodiálise de um hospital privado. 2006. [citado em: 26 mar 2009]. Disponível em: http://www.inicepg.univap.br/ INIC_2006/inic/inic/03/INIC0000608_OK.pdf.

6. Lo-Biondo G, Wood J. Pesquisa em enfermagem - métodos, avaliação e crítica. Rio de Janeiro: Guanabara-Koogan; 2001.

7. Lata AGB, Albulquerque IG, Carvalho LASBP, Lira ALBC. Diagnósticos de enfermagem em adultos em tratamento de hemodiálise. Acta Paul Enferm 2008; 2 I (esp): 160-3.

8. North American Nursing Diagnosis Association (NANDA). Diagnósticos de enfermagem: definições e classificação - 20072008. Porto Alegre: Artmed; 2008.

9. Mendonça RR, Lima LR. Perfil epidemiológico do paciente renal crônico em tratamento hemodialítico em Anápolis-GO. Interseção 2008; 2(2):29-36.

10. Godinho TM, Lyra TG, Braga PS, Queiroz RA, Kraychete AC, Gusmão ENA, Lopes AA, Rocha PN. Perfil do paciente Que inicia hemodiálise de manutenção em hospital público em Salvador Bahia. J Bras Nefrol 2006;; 28(2): 96-103.

11. Sociedade Brasileira de Nefrologia. Sociedade Brasileira de Enfermagem em Nefrologia. Associação de pacientes Renais Crônicos. Perfil da doença renal crônica: o desafio brasileiro. São Paulo; 2007. [citado em: 10 ago 2009]. Disponível em: http://ww2.prefeitura.sp.gov.br//arQuivos/secretarias/saude/ programas/0007/Doenca_Renal_Cronica.pdf
12. Bastos MG, Carmo WB, Abrita RR, Almeida EC, Mafra D, Costa DMN, et al. Doença renal crônica: problemas e soluções. I Bras Nefrol 2004; 26(4): 203-15.

13. Costa IAC, Vieira-Neto OM, Moysés Neto M. Insuficiência renal aguda. Medicina 2004; 36: 307-24.

14. Leal VO, Leite Júnior M, Mafra D. Acidose metabólica na Doença Renal Crônica: abordagem nutricional. Rev Nutrição 2008; 21(1): 93-103.

15. Abensur H, Bastos MG, Canziani MEF. Aspectos atuais da anemia na doença renal crônica. J Bras Nefrol 2006; 28(2): I04-7.

16. Ramos IC, Queiroz MVO, Jorge MSB. Cuidado em situação de Doença Renal Crônica: representações sociais elaboradas por adolescentes. Rev Bras Enferm 2008; 6I (2): 193-200.

17. Valenzuela RGV, Giffoni AG, Cuppari L, Canziani MAF. Estado nutricional de pacientes com insuficiência renal crônica em hemodiálise no Amazonas. Rev Assoc Med Bras 2003; 49(I): $72-8$.

18. Morsch C, Gonçalves LF, Barros E. Índice de gravidade da doença renal, indicadores assistenciais e mortalidade em pacientes em hemodiálise. Rev Assoc Med Bras 2005; 5 I (5): 296-300.

19. Martins MRI, Cesarino CB. Qualidade de vida de pessoas com Doença Renal Crônica em tratamento hemodialítico. Rev LatinoAm Enfermagem 2005; 13(6): 670-6.

20. Castro M, Caiuby AVS, Draibe AS, Canziani MEF. Qualidade de vida de pacientes com insuficiência renal crônica em hemodiálise avaliada através do instrumento genérico SF-36. Rev Assoc Med Bras 2003; 49(3): 245-9.

21. Kusumota L, Rodrigues RAP, Marques S. Idosos com insuficiência renal crônica: alterações do estado de saúde. Rev Latino-Am Enfermagem 2004; I2(3): 525-32. 